\title{
ENERGY LOSS and ANGULAR DISTRIBUTIONS of GOLD CLUSTER CONSTITUENTS
}

\author{
S. BOUNEAU ${ }^{1}$, A. BRUNELLE ${ }^{1 *}$, S. DELLA-NEGRA ${ }^{1}$, J. DEPAUW ${ }^{1}$, \\ D. JACQUET ${ }^{1}$, Y. LE BEYEC ${ }^{1}$, A. NOVIKOV ${ }^{1}$, M. PAUTRAT ${ }^{1}$ \\ and H.H. ANDERSEN ${ }^{2}$ \\ ${ }^{1}$ Institut de Physique Nucléaire, $\mathrm{IN}_{2} \mathrm{P}_{3}$-CNRS, 91406 Orsay, France \\ ${ }^{2}$ Niels Bohr Institute, Blegdamsvej 17, DK-2100 Copenhagen Ø, Denmark
}

November 16, 2006

\begin{abstract}
Heavy gold cluster beams are accelerated to high energy (hundreds of keV/atom) and break up when going through a thin foil. The energy and angular distributions of the constituents are then measured and very well reproduced by a SRIM code calculation, which takes into account atomic interactions only. These distributions do not depend on the number of constituents in the cluster and are found to be the same as those of single gold atoms at the same velocity, in the studied energy range.
\end{abstract}

\section{Introduction}

Non linear physical processes are frequently observed when polyatomic projectiles interact with solid materials. The enhancement of the sputtering and secondary ion emission yields, due to small polyatomic ions (dimers,trimers), with respect to single atoms, has been known for a long time as reviewed in [1]. However, a wider range of cluster sizes and energies, $\mathrm{Au}_{n}$ clusters in the

*Present address : ICSN-CNRS, Avenue de la Terrasse F 91198 Gif-sur-Yvette 


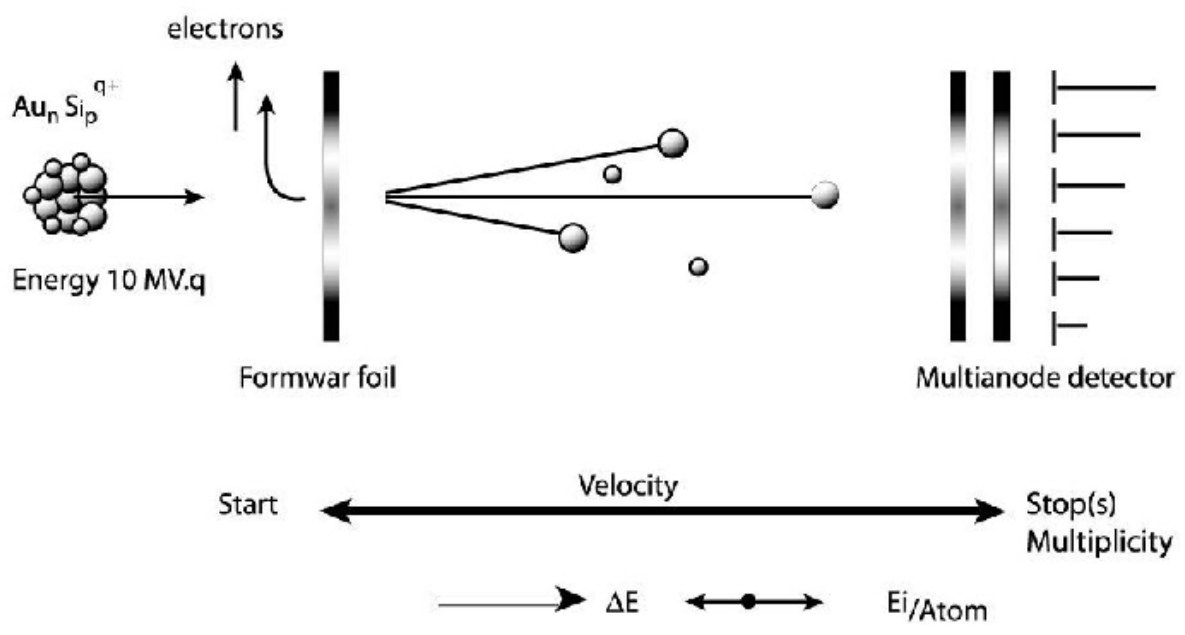

Figure 1: Schematic view of the experimental set-up.

$\mathrm{keV}$ to $\mathrm{MeV}$ energy/atom range, has allowed to analyse such effects in more detail. The sputtering yields due to $\mathrm{Au}_{n}$ clusters $(1 \leq \mathrm{n} \leq 13)$ on metallic targets (silver and gold) vary roughly as $\mathrm{n}^{2}$, at a given projectile velocity, and the maximum is found around $200-250 \mathrm{keV} /$ atom $[2,3]$ i.e. somewhat below the maximum in the nuclear stopping $\mathrm{S}_{n}$. The secondary ion emission yields, from organic and inorganic targets irradiated by $\mathrm{Au}_{n}$ beams $(\mathrm{n}=1$ to 4 ), scale also as $\mathrm{n}^{2}$, at a given velocity, with a maximum at some tens of $\mathrm{keV}$ per atom [4]. A very large effect of the $\mathrm{n}$ parameter on the measured secondary ion emission yields was observed also for bio-organic targets made of peptides [5,6], at an acceleration of $10 \mathrm{kV}$ per charge.

On the contrary, the secondary electron emission from solid surfaces, bombarded by $\mathrm{Au}_{n}$ cluster beams $(1 \leq \mathrm{n} \leq 5)$ between $33 \mathrm{keV}$ and $6 \mathrm{MeV}$ per atom, follows a sublinear law in $\mathrm{n}^{0.6}$, the sublinear effect increasing with the energy per atom in the cluster [7].

In this work gold cluster $\mathrm{Au}_{n}^{q+}$ beams, with $\frac{n}{q}$ ratios from 1 to 40, are accelerated by the M.P. Tandem in Orsay at energies between 1 and 10 $\mathrm{MeV}$ per charge. The present aim is to look for the possibility of other non linear behaviours and, in particular, to study the cluster size influence on the energy loss process and on the angular distributions of the constituents, when the beam passes through a thin amorphous polymer foil (Formvar). In this energy region the nuclear stopping power $\mathrm{S}_{n}$ dominates the electronic one. 
If $\mathrm{S}_{t}$ is the total stopping power, for gold atoms in Formvar the ratio $\frac{S_{n}}{S_{t}}$ is equal to $77 \%$ at $200 \mathrm{keV}$ and to $52 \%$ at $2 \mathrm{MeV}$. Clusters with an $\frac{n}{q}$ ratio of 5 , 7, 9, 27 and 40 are used. The time-of-flight, depending on the energy loss in the foil, and the angular distributions of the constituents are measured with a multipixel detector and compared to the results of SRIM code simulations and, for the angular distributions, to analytical calculations.

\section{Experimental methods}

The production of the cluster beams at the M.P. Tandem, the experimental set-up (shown in Fig. 1) and the mass and charge identification of the clusters have been discussed in detail in [8].

Table 1: Experimental conditions

\begin{tabular}{c|c|c|c|c|c}
$\frac{n}{q}$ & $\begin{array}{c}\text { Energy/Au } \\
\mathrm{MeV}\end{array}$ & $\begin{array}{c}\text { Formvar foil } \\
\text { thickness }(\mathrm{nm})\end{array}$ & Detector & $\bar{q}$ & $\bar{n}$ \\
\hline 5 & 2.008 & $49 \pm 2$ & $\mathrm{D} 64$ & 1 & 5 \\
7 & 1.434 & - & - & 1 & 7 \\
9 & 1.115 & - & - & 1 & 9 \\
27 & 0.372 & - & - & 3 & 81 \\
5 & 1.808 & $25 \pm 2$ & $\mathrm{D} 256$ & 1 & 5 \\
40 & 0.226 & - & - & 3.4 & 136 \\
9 & & & & & \\
27 & 0.2 & $26 \pm 2$ & $\mathrm{D} 256$ & 1.8 & 16 \\
40 & 0.2 & - & - & 3.6 & 97 \\
& 0.2 & - & - & 4.6 & 184
\end{tabular}

The cluster beams studied here are accelerated by a potential $\mathrm{V}(1 \leq \mathrm{V}$ $\leq 10 \mathrm{MV}$ ) and the energy of their atomic fragments is $\mathrm{E}_{i}=\frac{V * q}{n} \mathrm{MeV}$. In a series of these experiments, the potential has been adjusted to make the energy per gold atom the same for the $\mathrm{Au}_{9}^{+}, \mathrm{Au}_{27 q}^{q+}$ and $\mathrm{Au}_{40 q}^{q+}$ clusters (see Table 1). An angular deflection of $1.29^{\circ}$ through the analysing magnet confirms the $\frac{n}{q}$ ratio selection of the Wien filter, located together with the ion 
source, in the accelerator terminal. It is worth recalling that for each $\frac{n}{q}$ value, there is a mass and charge distribution getting broader as $\frac{n}{q}$ increases. The mean charge, $\bar{q}$, and number of constituents, $\bar{n}$, are shown in Table 1 ; in [8], $\bar{n}$ is deduced from the measured multiplicity distributions and corresponds to a $\bar{q}$ value through the $\mathrm{n} / \mathrm{q}$ ratio. The apparent discrepancies, for the same $\frac{n}{q}$, are due to different ion source conditions [8].

The projectiles are fragmented when passing perpendicularly through a Formvar foil of known thickness. Various foils of known, different thickness are used, their thickness being measured by the energy loss of $\alpha$ particles. To investigate a possible presence of pinholes a careful scanning of the foils by the beam, in both directions, is performed to detect a corresponding shift of the time-of-flight peak but none was observed. For this control the beam diameter and bin size are $0.3 \mathrm{~mm}$. The exiting atoms are then detected by a micro-channel plate and multianode detector, of the type described in $[8,9]$, which enables to measure the number of constituents, their time-of-flight and angular distributions. Two of these detectors are used, D256 and D64, having respectively a 256 and a 64 pixel anode array.

The start of the time-of-flight measurements is given by the electron (or $\mathrm{H}^{-}$) emission induced by the projectiles passing through the Formvar foil. The angular distributions of the ions coming out of the foil can be deduced from the two-dimensional position spectra, delivered by the multipixel detector, in coincidence with windows set on the time-of-flight peaks. These two conditions represent a guarantee against the influence of pinholes, if there were any present, otherwise undetected because too tiny.

\section{Experimental results}

The experimental time-of-flight spectrum, obtained for $\frac{n}{q}=40$, is given in Fig. 2. In this case the clusters, with a total energy of $9.04 \mathrm{MeV}$, go through a $25 \mathrm{~nm}$ Formvar foil and the fragments are detected by the D256 detector. As can be seen in Fig. 2, two peaks are present in the time-of-flight spectrum. The main one, in agreement with the energy/atom of the projectiles, is due to gold atoms, the second one corresponds to a speed about twice that of the first one and comes, as shown below, from the ejection of light ions like $\mathrm{H}^{+}$and $\mathrm{C}^{+}$from the target. The corresponding angular distributions are displayed in Fig. 3. The central cuts of the two-dimensional position spectra, recorded by the detector, are obtained by setting gates on the two time-of- flight peaks. The gold ions look properly centered, but the maximum 
intensity for the light ions is located on a ring, about 5 pixels from the centre, which is compatible with an ejection cone. The mean radius of this ring gives the ejection angle of the light ions : $\theta=3^{\circ}$. In both cases the distribution is truncated by the detector solid angle $\Omega$.

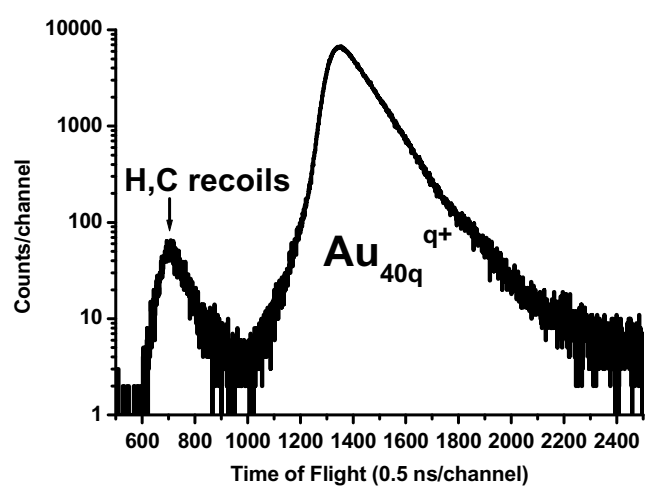

Figure 2: Time-of-flight spectrum of the cluster constituents from $\mathrm{Au}_{40 q}^{q+}$ at $226 \mathrm{keV} / \mathrm{Au}$, in the detector solid angle.

The most probable light ion ejected is $\mathrm{H}^{+}[10]$, probably followed by $\mathrm{C}^{+}$. The time-of-flight expected for these ions may be computed and compared to the measured value. The energy of the ejected light ion is $\mathrm{E}_{L I}$ so that :

$$
\mathrm{E}_{L I}=\mathrm{E}_{A u} \cos ^{2} \theta \frac{4 m M}{(m+M)^{2}}
$$

$\mathrm{m}$ and $\mathrm{M}$ being the light ion and gold masses respectively and $\theta$ the ejection angle of the light ion. From this energy, it is possible to estimate the light ion velocities and time-of-flights, depending on their emission point at the entrance of or exit from the target. The calculated time-of-flights, for $\mathrm{H}^{+}$ and $\mathrm{C}^{+}$mixed up in the spectrum, go from 312 to $345 \mathrm{~ns}$, in good agreement with the $339 \pm 17$ ns experimental value. Thus the position spectra and timeof-flight measurements confirm the assumption that light ions are recoiled from the target by the beam.

For $\mathrm{Au}_{n q}^{q+}$ clusters with $\mathrm{n}=9,27,40$, at the same velocity, the ejected light ion rate increases proportionally to the mean number of constituents.

Gold cluster constituents have the same time-of-flight whatever their number, if the parent clusters have the same velocity. This is illustrated 

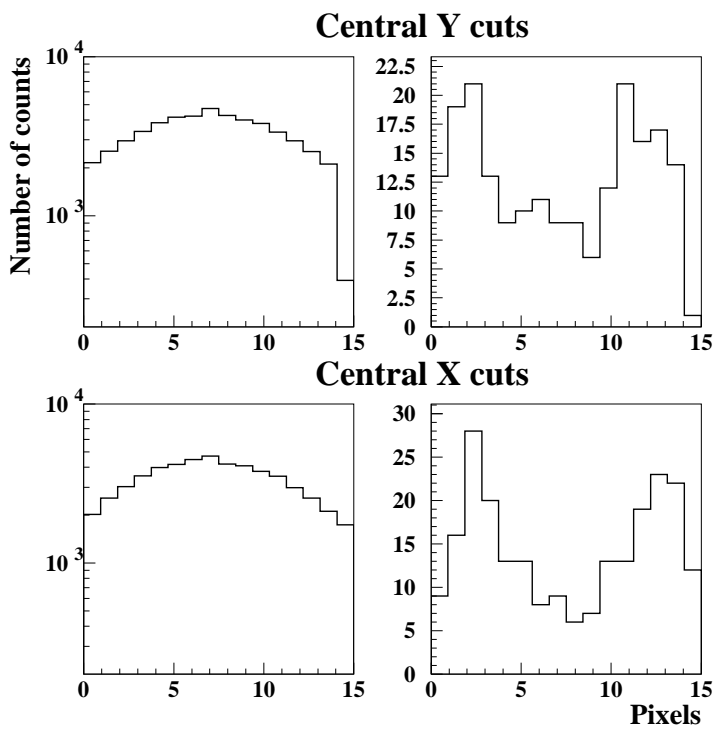

Figure 3: Central $\mathrm{X}$ and $\mathrm{Y}$ sections of the two-dimensional distributions registered by the D256 detector for gold (right) and light (left) ions, from $\mathrm{Au}_{40 q}^{q+}$ clusters at $226 \mathrm{keV} / \mathrm{Au}$.

in Fig. 4 showing the time-of-flight spectra of the constituents from $\mathrm{Au}_{9}^{+}$, $\mathrm{Au}_{27 q}^{q+}$ and $\mathrm{Au}_{40 q}^{q+}$ at $200 \mathrm{keV} / \mathrm{Au}$. The time-of-flight values differ by $2.5 \%$, i.e. they agree within the experimental errors. In Fig. 5 are displayed the $\mathrm{Y}$ cuts of the correponding two-dimensional position spectra (it is possible to neglect the variation of the solid angle $\Omega$ as it is only $0.6 \%$ between the center and the edges of the detector). The similarity of the three curve shapes is remarkable, with the same half width at half maximum of $53 \pm 3 \mathrm{mrad}$ . From the time-of-flight and angular distribution measurements no cluster effect can be claimed.

The only and very interesting difference, in the angular distribution spectra, concerns the central pixel, where the number of counts exceeds that expected from the interpolation between the neighbouring pixels, although the difference remains very small because of the small solid angle covered. The number of this kind of events, increases with the cluster projectile mass, at a given velocity. This could be related to a clearing-the-way effect, as described in [11], so that a proportion of undeflected atoms, depending on the number $n$ of cluster constituents, can be observed. From our results, however, we can only deduce that the rate of these events, of the order of a few per cent, is proportionnal to a power of $\mathrm{n}, \mathrm{n}^{\alpha}$ with $\alpha<1$. The energy 


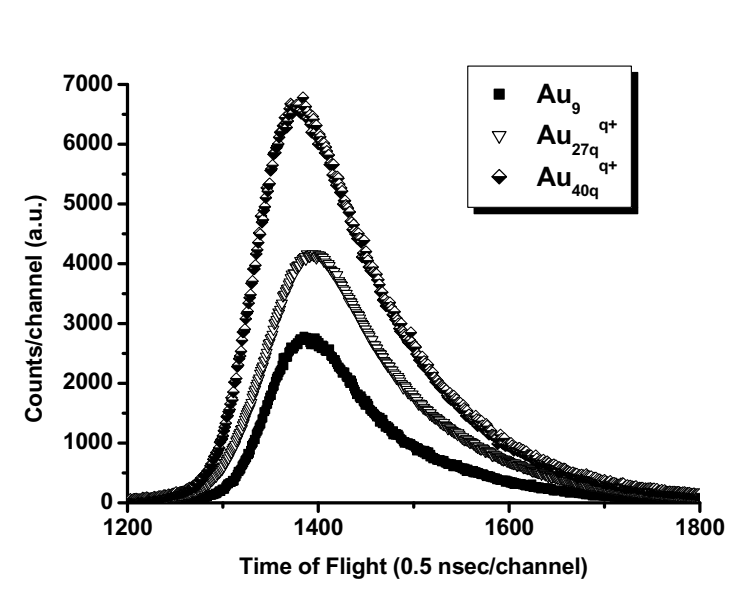

Figure 4: Time-of-flight spectra of the cluster constituents from $\mathrm{Au}_{9}^{+}$, $\mathrm{Au}_{27 q}^{q+}$ and $\mathrm{Au}_{40 q}^{q+}$ at $200 \mathrm{keV} /$ atom.

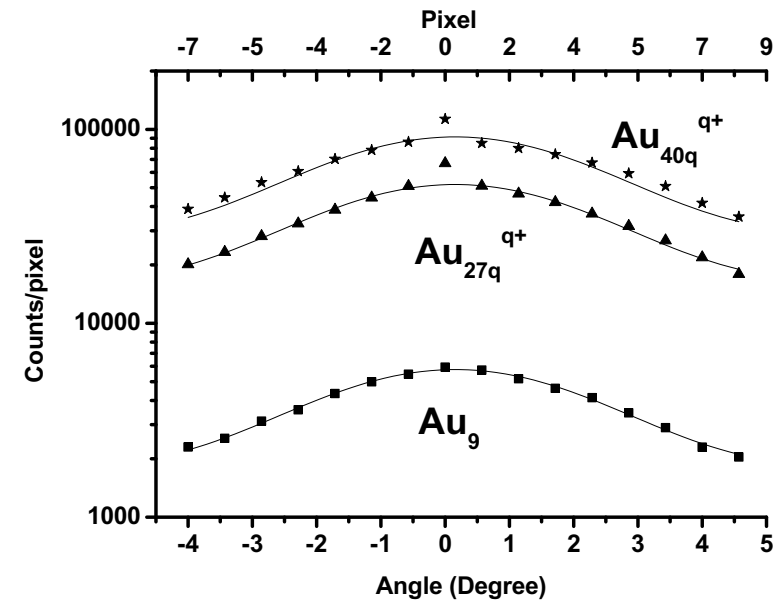

Figure 5: Angular distributions, in the central X plane, for the cluster constituents from $\mathrm{Au}_{9}^{+}, \mathrm{Au}_{27 q}^{q+}$ and $\mathrm{Au}_{40 q}^{q+}$ at $200 \mathrm{keV} /$ atom (D256 detector).

loss for the central pixel was found not to be significantly lower than for the surrounding pixels.

\section{Discussion}

The energy and angular distributions, after passage through the Formvar foil, are compared to the results of a SRIM code [12] simulation. A more detailed analysis of the angular distributions is also performed.

\section{a) Experiment and simulation}

The SRIM code deals with atomic projectiles; it's use to simulate the cluster interaction with a Formvar foil assumes that this interaction is similar to that of a single atomic ion. We shall see that this assumption is confirmed by experiment.

The energy distributions are related to the time-of-flight ones. Hereafter a convolution of the time-of-flight and angular distributions, calculated by SRIM, with a Gaussian distribution, accounting for the time-of-flight resolving power and the inhomogeneity of the Formvar foil, is used and compared to the experimental results. 

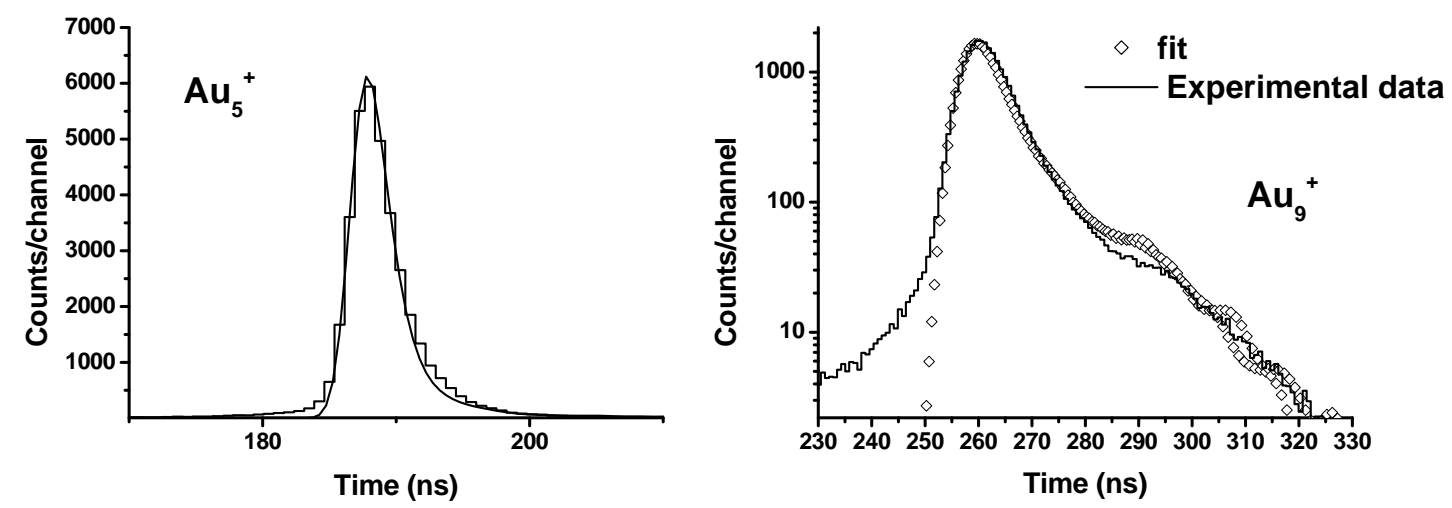

Figure 6: Experimental and SRIM simulated (see text) time-of-flight spectra for the $\mathrm{Au}_{5}^{+}$(histogram $=$experiment) and $\mathrm{Au}_{9}^{+}$projectiles.

The time-of-flight spectra of Fig. 6, for the $\mathrm{Au}_{5}^{+}$and $\mathrm{Au}_{9}^{+}$projectiles at a total energy of $10.04 \mathrm{MeV}$, are well reproduced by such a calculation, with a standard deviation of $\sigma=3.8$ ns for the Gaussian distribution. In Fig. 7 the experimental and calculated angular distributions are shown for $\mathrm{Au}_{5}^{+}$ clusters, in the same energy region, after passing through two Formvar foils of different thicknesses, the detectors being also different. The $25 \mathrm{~nm}$ thick foil is associated to the D256 detector and the 49nm one to the D64 detector. In both cases the data are well fitted by the simulation, the thicker target corresponding, as expected, to a broader curve.
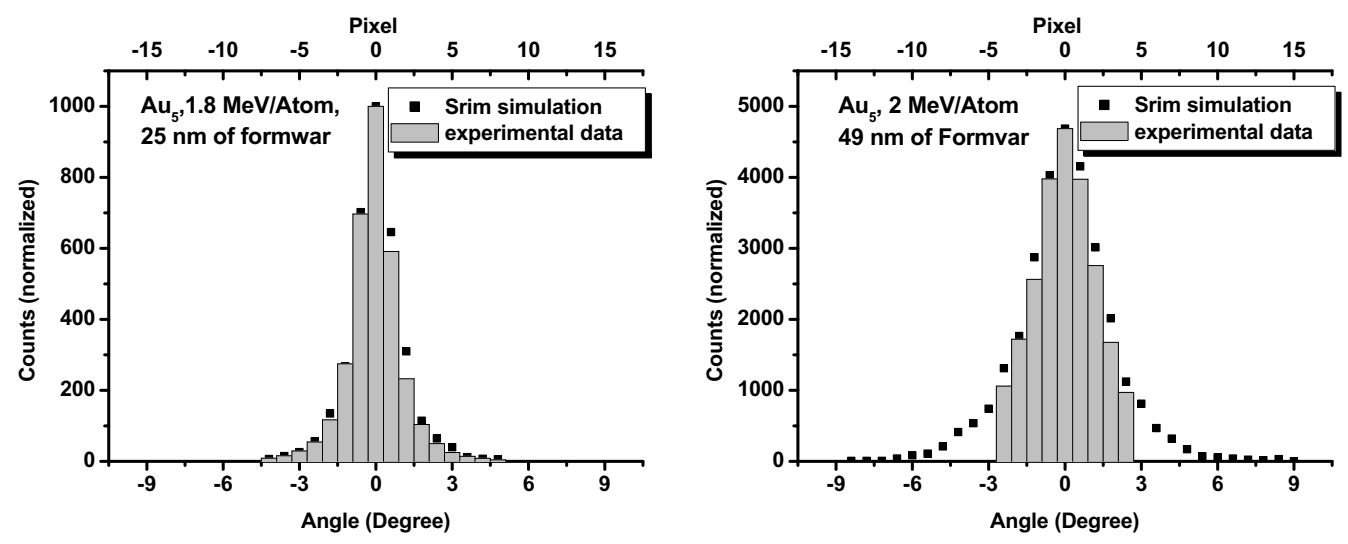

Figure 7: Central Y cuts of the two-dimensional position spectra for $\mathrm{Au}_{5}^{+}$ constituents, compared to the SRIM simulation, for two Formvar foil thicknesses. 

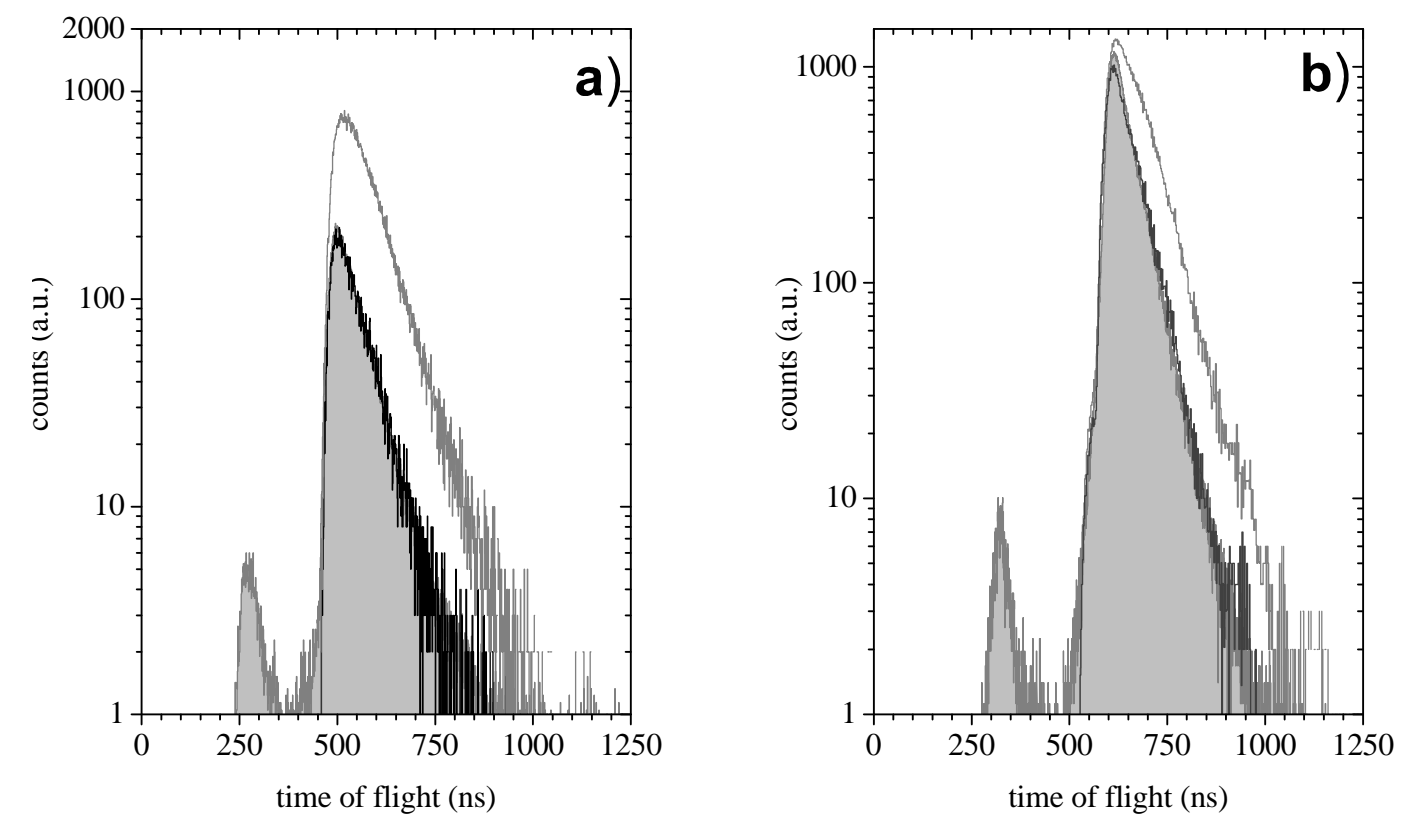

Figure 8: Time-of-flight spectra associated to the projectiles with a) $\frac{n}{q}=$ 27 (10.04 MeV, D64) and b) $\frac{n}{q}=40(9.04 \mathrm{MeV}, \mathrm{D} 256)$. The experimental spectra are in full grey, the total simulation in grey, and the simulation inside $\Omega$ in black.

Using the Fig. 6 parameters gives the result of Fig. 8a for the time-offlight of $\mathrm{Au}_{27 q}^{q+}$ at 10.04.q MeV, the Formvar foil and detector being the same. However, also the $\mathrm{Au}_{26}^{+}$and $\mathrm{Au}_{28}^{+}$mass contributions have been introduced, in agreement with the mass separation of the Wien filter $\left(\frac{m}{\Delta m}=20\right)$. The $\mathrm{Au}$ clusters with $\mathrm{n}=51$ to 57 , corresponding to a $2^{+}$charge, when included, do not appreciably change the time-of-flight spectrum. The width of the time-of-flight distribution for each mass and the shift of their centroids make it impossible to deduce the existence of charges $\mathrm{q} \geq 3$ from the experimental spectrum.

The same comparison between experimental data and SRIM computation for $\mathrm{Au}_{40 q}^{q+}$ is given in Fig. 8b. This fit also takes into account the mass distribution, due to our limited mass separation, and the detector solid angle; without any other parameter the time-of-flight spectrum is perfectly repro- 
duced, for a thinner target and a 9.04.q MeV energy.

In Fig. 8 are also drawn the time-of-flight distributions without the limitation of the detection solid angle $\Omega$. These curves, but also those of Fig. 9, show that this truncation reproduces correctly the experimental results. In Fig. 9 the central Y cut of the two-dimensional position spectrum, simulated by SRIM, is compared to the experimental one, in the case of $\mathrm{Au}_{40 q}^{q+}$ for the D256 detector. The two curves are in good agreement but the experimental count loss, due to the detector solid angle, is clearly visible in this figure.

The solid lines drawn in Fig. 5 are the result of the SRIM based simulation which reproduce the angular distribution data for different cluster masses.

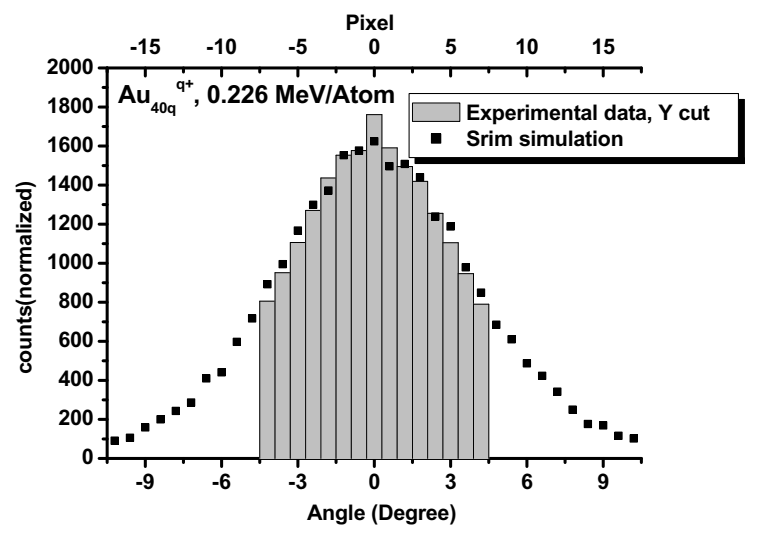

Figure 9: Experimental central Y cut of the two-dimensional position spectrum for the $\mathrm{Au}_{40 q}^{q+}$ constituents compared to the SRIM simulation (D256 detector).

From $\mathrm{Au}_{5}^{+}$to $\mathrm{Au}_{40 q}^{q+}$, passing through a Formvar film, no difference is observed between the experimental time-of-flights, or angular distributions, of the constituents and those computed with the SRIM code, for all the corresponding energies per atom. The experiments performed with several clusters like $\mathrm{Au}_{9 q}^{q+}, \mathrm{Au}_{27 q}^{q+}$ and $\mathrm{Au}_{40 q}^{q+}$, with the same energy per atom, i.e. the same speed, lead to the same time-of-flights, pointing clearly to a lack of difference in the energy loss measurements when the number of constituents increases up to a few hundreds. The angular distributions, in this case, are also identical which implies identical nuclear stopping powers. These results confirm and extend the conclusion of Ref. [13] which describes the analysis of projected ranges for gold clusters in $\mathrm{Al}, \mathrm{Si}$ and $\mathrm{Cu}$ targets.

In view of the lack of non-linear effects in the nuclear stopping found above it is surprising to see He et al. [14] claim substantial non-linearities for 
$0.9 \mathrm{MeV} / \mathrm{Al} \mathrm{Al}_{3}$ clusters in $\mathrm{Si}$ with a Ta marker. They studied the mixing of the said marker and assume a priori that the square of the half- width of the

depth distribution of the Ta marker is proportional to the locally deposited energy, which again is taken proportional to the nuclear stopping power, i.e. they assume linearity between the nuclear stopping power and the mixing. From their data, a $15 \%$ average enhancement over $25 \mathrm{~nm}$ is deduced. As our clusters are substantially larger than theirs and have a much higher $\mathrm{Z}_{1}$, and as our energies are closer to the maximum in $\mathrm{S}_{n}$ than theirs, we should have seen very substantial non-linear effects in $S_{n}$. From our lack of any such evidence and from the theoretical [11] and experimental [15] evidence for such effects only to exist at very low energies and having the opposite sign of what was found by He et al., we think that their interpretation is not correct. Their results can be explained, in agreement with the others, by the observation of a non-linear relation between deposited energy density and ion beam mixing, without a non-linearity in $\mathrm{S}_{n}$.

\section{b) Quantitative analysis of the angular distributions.}

The aim here is to compare the preceeding data with the results of an analytical calculation, based on Meyer's theory [16] revised by Sigmund and Winterbon [17], which leads to a "universal" relation between the reduced target thickness :

$$
\tau=\pi a^{2} N t
$$

and the reduced scattering angle :

$$
\tilde{\alpha}=\frac{E a}{2 Z_{1} Z_{2} e^{2}} \alpha
$$

a being the screening parameter, $\mathrm{N}$ the target atomic density, $\mathrm{t}$ the target thickness, E the projectile energy, $\mathrm{Z}_{1}$ and $\mathrm{Z}_{2}$ the projectile and target atomic numbers, -e the electron charge and $\alpha$ the laboratory scattering angle. The screening parameter taken, in most cases (see [16] for example), is the Lindhard value $[18]$ :

$$
\mathrm{a}=0.885 \mathrm{a}_{0}\left(Z_{1}^{2 / 3}+Z_{2}^{2 / 3}\right)^{-1 / 2}
$$

$\mathrm{a}_{0}=0.52910^{-8} \mathrm{~cm}$ being the first Bohr radius.

The reduced half-widths $\tilde{\alpha}_{1 / 2}$ plotted as a function of $\tau$ for different multiple scattering distributions fall then on a single curve, independent of $Z_{1}$, 
Table 2: Comparison between experimental, calculated and simulated halfwidths

\begin{tabular}{clcllll}
$\frac{n}{q}$ & $\begin{array}{l}\text { Energy/Au } \\
\text { MeV }\end{array}$ & $\begin{array}{c}\text { Formvar foil } \\
\text { thickness }\end{array}$ & $\begin{array}{l}\alpha_{1 / 2} \text { exp. }(\mathrm{mrad}) \\
\text { truncated }\end{array}$ & $\begin{array}{l}\alpha_{1 / 2} \text { th. } \\
(\mathrm{mrad})\end{array}$ & $\begin{array}{l}\alpha_{1 / 2} \mathrm{SRIM}(\mathrm{mrad}) \\
\text { truncated }\end{array}$ & $\begin{array}{l}\alpha_{1 / 2} \mathrm{SRIM}(\mathrm{mrad}) \text { total } \\
1 / 2 \text { gaussians }\end{array}$ \\
\hline 5 & 2.008 & $49 \pm 2 \mathrm{~nm}$ & $19.8 \pm 0.6$ & 24.7 & 19.3 & $27.7 / 19.3(41 \%)$ and $44.4(59 \%)$ \\
5 & 1.808 & $25 \pm 2 \mathrm{~nm}$ & $13.4 \pm 0.6$ & 13.3 & & $14.2 / 10.7(53 \%)$ and $26.7(47 \%)$ \\
40 & 0.226 & $25 \pm 2 \mathrm{~nm}$ & $64.8 \pm 2.9$ & 106 & $64.2 \pm 1.7$ & $77 \pm 1.8 / 59.5(40 \%)$ and $95.5(60 \%)$ \\
$9,27,40$ & 0.2 & $26 \pm 2 \mathrm{~nm}$ & $52.5 \pm 2.9$ & 130 & $69.5 \pm 1.7$ & $79.4 \pm 1.8 / 47.9(19 \%)$ and $91(81 \%)$ \\
$9,27,40$ & 0.2 & $20 \mathrm{~nm}$ & & 95 & $53 \pm 1.7$ & $66 \pm 1.7 / 48.2(35 \%)$ and $85.6(65 \%)$ \\
\hline
\end{tabular}

$\mathrm{Z}_{2}$ and E. In these conditions, when $\tau$ is known the curve gives the corresponding $\tilde{\alpha}_{1 / 2}$ value and the laboratory $\alpha_{1 / 2}$ th. angle can be deduced and compared to the measured and simulated ones.

However, as was underlined previously, the influence of the detector solid angle is important. To extract a more precise half-width from a distribution, truncated in most cases by the detector size, a Gaussian distribution has been fitted to the experimental or simulated points. It reproduces very well the data and leads to a standard deviation and to a half-width $\alpha_{1 / 2}$ exp. or $\alpha_{1 / 2}$ SRIM. The values obtained for $\alpha_{1 / 2}$ exp., $\alpha_{1 / 2}$ th., $\alpha_{1 / 2}$ SRIM for a truncated at the detection surface and for a total distribution are reported in Table 2. The uncertainties on the $\alpha_{1 / 2}$ exp. values are deduced from the comparison between the $\mathrm{X}$ and $\mathrm{Y}$ cuts or from the variation between two cuts neighbouring the centre. Those on the $\alpha_{1 / 2}$ SRIM values come from the comparison of random distributions extracted from sets of $10^{6}$ events.

In Table 2 the measured half-width for the $\frac{n}{q}=9,27$ and 40 cluster constituents, at $200 \mathrm{keV} / \mathrm{Au}$, is abnormally smaller than that for the $\frac{n}{q}=$ 40 , at $226 \mathrm{keV} / \mathrm{Au}$ one. This can be due to a local inhomogeneity or to an uncertainty on the Formvar foil thickness. The last line of Table 2 shows that a Formvar foil $20 \%$ thinner compensates this half-width anomaly in the simulation.

The SRIM simulation results of Table 2 reproduce quite well the experimental values when the same solid angle is analyzed. The half-width of the distribution increases when the energy per atom decreases. However, the $\alpha_{1 / 2}$ value given by the total SRIM simulation (without angular limitation) is 20 to $30 \%$ greater than the experimental one. This difference is due to an important contribution $(>50 \%)$ of events with a large solid angle. These can be accounted for by introducing a second Gaussian distribution, with a half-width between 1.5 and 2.5 times that of the main one, which improves 
the fit to simulated and, most likely, to experimental data. These half-widths and the corresponding percentages are given in the last column of Table 2, together with the half-width associated to a single Gaussian distribution.

The analytical calculation agrees with experiment for the high energies, when the whole angular distribution is detected. But an important difference, about a factor of two, is observed for low energy projectiles when only the central part of the experimental distribution is analyzed. In the comparison between the SRIM simulation and the analytical calculation the same difference appears, but the second Gaussian distribution, extracted from the simulation, is closer to the analytical calculation in terms of $\alpha_{1 / 2}$. No explanation is presently available for this difference. In the analytical calculation a decrease of the screening parameter, sometimes used for low energy interactions, could bring the calculated values closer to the experimental ones. Nevertheless, to carry on with this analysis would be uncertain because of the truncation of the experimental distributions and of the small variety of experimental conditions.

\section{Conclusion}

A variety of gold clusters, from $\mathrm{Au}_{5}^{+}$to $\mathrm{Au}_{40 q}^{q+}$, at different energies per atom, passing through Formvar foils 25 or $49 \mathrm{~nm}$ thick, have been used to study the time-of-flight and angular distribution spectra of the atomic constituents. It is shown that SRIM simulations of single atoms, at the same velocity, reproduce the experimental results obtained with clusters. It thus appears that, in the energy range $200 \mathrm{keV} \leq \mathrm{E} / \mathrm{Au} \leq 2 \mathrm{MeV}$, a $\mathrm{Au}_{n}$ cluster behaves as a group of $\mathrm{n}$ independent $\mathrm{Au}$ atoms, with respect to the energy loss and straggling processes. This is also true for the ejection of light ions from the target.

No clustering effects were seen for any of the direct projectile-target collisions studied here but strong non linear effects are observed for sputtering, secondary ion emission, radiation damage and mixing, that involve collision cascades. The energy transferred to the medium cannot account for the non linear effects observed with cluster projectiles; it is necessary to consider the energy density, for which the difference between clusters and atoms becomes appreciable. 


\section{References}

[1] H.H. Andersen in Fundamental Processes in Sputtering of Atoms and Molecules, edited by Peter Sigmund [Mat. Fys. Medd. K. Dan. Vid. Selsk 43(1993) 127]

[2] S. Bouneau, A. Brunelle, S. Della-Negra, J. Depauw, D. Jacquet, Y. Le Beyec, M. Pautrat, M.Fallavier, J.C. Poizat and H.H Andersen, Phys. Rev. B65 (2002) 144106.

[3] H.H Andersen, A. Brunelle, S. Della-Negra, J. Depauw, D. Jacquet, Y. Le Beyec, J. Chaumont and H. Bernas, Phys. Rev. Lett. 80 (1998) 5433.

[4] A. Brunelle, S. Della-Negra, J. Depauw, D. Jacquet, Y. Le Beyec, M. Pautrat, K. Baudin, H.H Andersen, Phys.Rev. A63 (2001) 022902.

[5] A. Tempez, J.A. Schultz, S. Della-Negra, J. Depauw, D. Jacquet, A. Novikov, Y. Le Beyec, M. Pautrat, M. Caroff, M. Ugarov, H. Bensaoula, M. Gonin, K. Fuhrer and A. Woods, Rapid Commun. Mass Spectrom. 18 (2004) 371.

[6] A. Novikov, M. Caroff, S. Della-Negra, J. Depauw, M. Fallavier, Y. Le Beyec, M. Pautrat, J.A. Schultz, A. Tempez, and A.S. Woods, Rapid Commun. Mass Spectrom. 19 (2005) 1851.

[7] K. Baudin A. Brunelle, S. Della-Negra, J. Depauw, Y. Le Beyec, E.S. Parilis, Nucl. Instr. and Meth. B117 (1996) 47.

[8] S. Bouneau, S. Della-Negra, J. Depauw, D. Jacquet, Y. Le Beyec, J.P. Mouffron, A. Novikov, M. Pautrat, Nucl. Instr. and Meth. B225 (2004) 579.

[9] S. Bouneau, P. Cohen, S. Della-Negra, D. Jacquet, Y. Le Beyec, J. Le Bris, M. Pautrat and R. Sellem, Rev. Scient. Instr. 74 (2003) 57.

[10] R. Bimbot, S. Della-Negra, D. Gardès. H. Gauvin et B. Tamain, Revue de Physique Appliquée 13 (1978) 393

[11] V.I. Shulga and P. Sigmund, Nucl. Inst. and Meth. B47 (1990) 236.

[12] SRIM 2000.39, www.SRIM.org.

For the background see : J.F. Ziegler, J.P. Biersack and U. Littmark, in Stopping Powers and Ranges of Ions in Matter, ed J.F. Ziegler (Pergamon Press, New York, 1985)

[13] H.H. Andersen, A. Johansen, M. Olsen, V. Touboltsev, Nucl. Instr. and Meth. B212 (2003) 56.

[14] W.-H. He, H.-S. Kang, D.-Y. Shen, R. Nie, Y.-X. Shen, F.-R. Ding, Nucl. Instr. and Meth. B201 (2003) 449.

[15] H.H. Andersen, J. Bøttiger, H. Knudsen, P. Møller Petersen and T. Wohlenberg, Phys. Rev. A10 (1974) 1568.

[16] L. Meyer, Phys. Stat. Sol. B44 (1971) 253. 
[17] P. Sigmund and K.B. Winterbon, Nucl. Inst. and Meth. 119 (1974) 541.

[18] J. Lindhard, V. Nielsen and M. Scharff, Mat. Fys. Medd. K. Dan. Vid. Selsk. 36 (1968) No. 10 